\title{
Curso de extensão de leitura crítica de artigos científicos: relato de experiência
}

Stefanie Griebeler Oliveira

Universidade Federal de Pelotas | Brasil

stefaniegriebeleroliveira@gmail.com

\section{Simone Coelho Amestoy}

Universidade Federal de Pelotas | Brasil simoneamestoy@hotmail.com

\section{Raquel Pötter Garcia}

Universidade Federal do Pampa | Brasil raquelpottergarcia@gmail.com

\section{Bianca Pozza dos Santos}

Universidade Federal de Pelotas | Brasil bi.santos@bol.com.br

\section{Patricia Mirapalheta Pereira}

Universidade Federal de Pelotas | Brasil patihepp@yahoo.com.br

\author{
Andrieli Daiane Zdanski de Souza \\ Universidade Federal de Pelotas | Brasil \\ andriele_zdanski@hotmail.com

\section{Julia Cardoso Parra} \\ Universidade Federal de Pelotas | Brasil \\ juliacardosoparra@gmail.com
}

\section{Silvia Francine Sartor}

Indiana University Bloomington | Estados Unidos | Universidade Federal de Pelotas | Brasil sii.sartor@hotmail.com

\section{Kimberly Larroque Velleda}

Universidade Federal de Pelotas | Brasil kimberlylaroque@yahoo.com.br

\section{Resumo}

Relata-se a experiência do Curso de Extensão de Leitura Crítica de Artigos Científicos, o qual possibilitou um espaço para discussão de leitura e interpretação de artigos da área de saúde junto a acadêmicos e profissionais de saúde. Nesse projeto, foram realizados sete encontros, sendo três presenciais, com a discussão de um artigo por subgrupo e, quatro virtuais, com disparadores para discussão em uma rede social. Destaca-se que o Curso possibilitou a instrumentalização de profissionais da saúde e acadêmicos para a leitura crítica de artigos científicos, o qual poderá ser aplicado na prática e qualificar a assistência em saúde.

\section{Palavras-chave}

Artigo de revista. Relações Comunidade-Instituição. Atividade de extensão. 


\section{Introdução}

O hábito de ler é necessário ao indivíduo, uma vez que proporciona a descoberta de um mundo novo e fascinante. Além disso, a leitura permite a inserção no meio social, caracterizando-o, portanto, como cidadão participante (CARDOSO; PELOZO, 2007). A leitura e a interpretação estão presentes em todas as áreas do conhecimento, por conseguinte, as informações se disseminam cada vez mais rápido e por diferentes meios de comunicação (BONA; DAMINELLI; OLIVEIRA, 2012).

É por meio da leitura de artigos científicos que a sociedade pode obter conhecimento dos resultados de um trabalho de pesquisa, e o que este representa para a coletividade (BROFMAN, 2012). Ademais, quando as informações são disponibilizadas eletronicamente favorecem a aproximação dos públicos acadêmicos dos não acadêmicos, possibilitando a comunicação da ciência e a sua popularização (TENOPIR; KING, 2001; VALERIO; PINHEIRO, 2008).

A ampliação dos conhecimentos científicos para além do espaço acadêmico, de acordo com Valério e Pinheiro (2008), caracteriza-se como uma forma de entrelaçamento de saberes e de fortalecimento da divulgação das pesquisas realizadas. No ensino superior é comum a circulação de artigos científicos. No entanto, se por um lado, tal gênero é bastante familiar aos professores universitários, por outro, ele é geralmente desconhecido pelos estudantes que ingressam em uma universidade (BERTOLUCI, 2009). Nesse sentido, pelo fato dos alunos estarem se deparando pela primeira vez com o fazer científico, uma das maiores dificuldades pode ser o desconhecimento de como se faz pesquisa e, assim, não saberem como devem interpretar as informações lidas. Dessa forma, a leitura de artigos permite o aprender a aprender e explorar o conhecimento oriundo de investigações científicas (MATENCIO, 2002).

Por meio de um texto escrito, os leitores têm a possibilidade de apreender informações que podem ser compreendidas de diversas maneiras. Portanto, acredita-se que cursos de leitura de artigos relacionados à área da saúde, tais como atividades de extensão, podem ser relevantes para a aproximação da academia com a comunidade, uma vez que esta se encontra na ponta dos serviços de saúde (Bona; Daminelli; Oliveira, 2012). Por se tratar de um projeto de extensão, conforme Resolução 04/2013, esse tipo de atividade deve ultrapassar os muros da universidade (UFPEL, 2013), fato que foi viabilizado pela inserção de profissionais da saúde, os quais necessitam muitas vezes da leitura de artigos científicos para a busca de informações que possam subsidiar e qualificar a suas atividades práticas.

Para os acadêmicos participantes, o curso favorece a aproximação com materiais científicos, os quais são fontes confiáveis de conhecimento e aprofundamento teórico. A 
Curso de extensão de leitura crítica de artigos científicos: relato de experiência

aproximação da academia com os serviços de saúde foi relevante para as discussões realizadas durante as atividades do curso, devido às diversas experiências e possibilidades de reflexão. Além disso, o curso emergiu como uma possibilidade de problematização, socialização do conhecimento e fortalecimento dos laços entre os profissionais da saúde e os acadêmicos, com vistas a diminuir a distância existente entre as instituições de ensino e os serviços de saúde, corroborando para integração de tais espaços de educação formal e permanente.

Diante disso, o Curso de Extensão de Leitura Crítica de Artigos Científicos foi organizado e ofertado, aliando a necessidade da criação de um espaço para a discussão sobre leitura de artigos e a proposta de extensão universitária. Para tanto, objetivou-se relatar a experiência da realização deste aperfeiçoamento, o qual possibilitou um espaço para discussão de leitura e interpretação de artigos da área da saúde junto a acadêmicos e profissionais de saúde.

\section{Metodologia}

Este trabalho caracteriza-se como um relato de experiência, a partir da realização de um projeto de extensão que visou à proposição de um espaço para discussão sobre leitura e interpretação de artigos científicos para acadêmicos e profissionais da área da saúde. 0 relato de experiência é uma ferramenta da pesquisa descritiva, que apresenta uma reflexão sobre uma ação ou um conjunto de ações que abordam uma situação vivenciada no âmbito profissional de interesse da comunidade científica (CAVALCANTE; LIMA, 2012).

Tal ação extensionista originou-se a partir da articulação de docentes, pós-graduandos e acadêmicos da Faculdade de Enfermagem de uma universidade pública localizada na Região Sul do Brasil. A atividade foi realizada na modalidade de curso no período de 31 de outubro a 12 de dezembro de 2013, com três encontros presenciais e quatro virtuais. Estes últimos ocorreram por meio do uso de uma rede social, totalizando 40 horas. Os encontros foram amparados pela metodologia problematizadora, a qual se caracteriza por ser uma ferramenta fundamental para a ação/reflexão (SILVA; ASSIS; GENTILE, 2005).

A divulgação do Curso de Extensão de Leitura Crítica de Artigos Científicos ocorreu 15 dias antes de sua data prevista para início, e para tanto, utilizaram-se cartazes e panfletos, propagados em diversos locais onde profissionais de saúde atuam e acadêmicos transitam, tais como: Secretaria Municipal de Saúde, compreendendo 50 Unidades Básicas de Saúde e oito Centros de Atenção Psicossocial; dois hospitais de grande porte, três hospitais de médio porte, além de um serviço de urgência e emergência; instituições de ensino superior e de ensino técnico, que possuem cursos da área de saúde. 
Curso de extensão de leitura crítica de artigos científicos: relato de experiência

As inscrições foram realizadas por e-mail, de modo a manter o contato com os inscritos, sendo disponibilizadas 50 vagas. Os três encontros presenciais foram realizados, mensalmente, em uma sala da faculdade, onde o projeto de extensão estava vinculado. Os encontros virtuais, A, B, C e D foram operacionalizados pela criação de um grupo do curso na rede social Facebook, selecionada por ser uma das mais utilizadas na atualidade e também por possibilitar postagens nas quais outras pessoas possam comentar concomitantemente.

De maneira semelhante aos encontros presenciais, os encontros virtuais podem construir ambientes formais propícios e confortáveis às discussões, debates e formação de conceitos. Porém, somente nos encontros presenciais são criados ambientes informais em que erros, brincadeiras, invenções e discussões menos formais são mais naturais, pois esses não contam com mecanismos inibidores, tais como os registros completos de suas conversas (CARVALHO; RAMOS, 2007).

Em cada encontro presencial, com duração de quatro horas, foi discutido um artigo por grupo. Tais artigos foram selecionados previamente pelos integrantes da comissão organizadora, atentando para evitar repetição nas temáticas. No que tange aos critérios, preconizava-se artigos que tivessem elementos que apresentavam diferenciações e/ou problemas nos seus conteúdos principais (título - curto/extenso; resumo - estruturado, não estruturado; descritores - incompletos, completos; método - quantitativo/qualitativo; resultados e discussão - juntos, separados; conclusão/considerações finais - quantitativo/qualitativo; referências - atualizadas/desatualizadas). Buscou-se desta forma, ampliar o olhar dos participantes tanto para os elementos que compõem a estrutura de um artigo científico como para o conteúdo apresentado e sua qualidade.

Nos encontros virtuais, foram utilizados disparadores para discussão. Esses disparadores envolveram partes que compõe um artigo científico:: Introdução/Objetivos, Metodologia, Resultados e Discussão e/ou Conclusão. Os critérios de seleção para tais artigos foram os mesmos utilizados para os encontros presenciais.

Ao final de cada encontro presencial foi entregue uma ficha de avaliação da atividade proposta no curso que continha os itens: Excelente, Bom, Razoável e Ruim. Os quesitos avaliados foram os seguintes: Atendimento aos participantes antes de cada encontro; Divulgação (clareza, inscrição); Instalações físicas (acesso, sala); Identificação dos locais e informação fornecida; Equipamentos (multimídia e som); Apresentação dos temas programados; Comunicação, objetividade e clareza dos facilitadores e encontros; Temas abordados e atividades desenvolvidas no encontro; Atendimento aos participantes para esclarecimentos de uso do grupo na rede social; e Qualidade dos disparadores e clareza na explicação das atividades 
Curso de extensão de leitura crítica de artigos científicos: relato de experiência

propostas às discussões realizadas em forma de comentários. Ainda, havia um espaço disponível para observações e sugestões para o próximo encontro.

\section{Resultados}

Em aproximadamente duas semanas de abertura das inscrições para o Curso de Extensão de Leitura Crítica de Artigos Científicos, as 50 vagas ofertadas foram preenchidas. No entanto, em média, 31 participantes foram assíduos. Desses, seis profissionais de saúde e os demais acadêmicos.

No primeiro encontro presencial, realizado no dia 31 de outubro de 2013, houve a participação de 31 inscritos, sendo 11 profissionais de saúde e 20 acadêmicos. Na ocasião, foram discutidos, primeiramente, os objetivos do curso e, em um segundo momento, foi proposta a formação de pequenos grupos para facilitar a discussão, realizar a leitura de um artigo científico e elaborar uma lista de critérios, em que os participantes entendiam como importantes na leitura de um artigo.

Após a elaboração dessa lista, cada grupo expôs os critérios listados por meio de cartazes para os demais participantes do curso. Ao se tratar desses critérios, os mais apontados foram: título, que deve ser claro, objetivo, sucinto, corresponder com o que é abordado e atraente para a leitura do texto na íntegra; ano de publicação, levando-se em consideração a atualização; resumo, de modo a focar no objetivo do artigo, abordar resultados e conclusões, sendo claro, breve e com dados informativos; metodologia composta pela caracterização do estudo, a apresentação dos participantes, o local da pesquisa, o período, o referencial metodológico utilizado e os princípios éticos.

Durante a explanação dos grupos, os facilitadores do curso faziam também contribuições, esclarecendo dúvidas e destacando algumas potencialidades de cada item dos artigos abordados. No final desse primeiro encontro, foi entregue uma ficha de avaliação, em que grande parte dos participantes avaliou como Excelente ou Muito Bom os quesitos de organização, equipamentos e encontro com os assuntos propostos.

Quanto aos encontros virtuais, foram realizados por meio da publicação de um disparador de discussão. Os participantes puderam fazer comentários sobre a estrutura e o conteúdo da seção escolhida que compõem o artigo e os facilitadores compartilharam ideias por meio de comentários. Entre os dias 08 a 15 e 16 a 27 de novembro, respectivamente, ocorreram os encontros virtuais A e B. No encontro virtual A participaram 36 pessoas, sendo dez profissionais e os demais acadêmicos, comentando e/ou curtindo a atividade. 
Curso de extensão de leitura crítica de artigos científicos: relato de experiência

Utilizou-se o fragmento de um artigo composto por título, autores e resumo, para que os participantes respondessem às questões relacionadas com os seguintes assuntos: adequação do título; relevância do tema e sua inserção na atualidade, por meio da realização de uma breve busca para averiguar quantos artigos eram encontrados sobre o assunto no último ano; os participantes deveriam também verificar se os autores do artigo eram especialistas no tema, realizando a conferência por meio do currículo Lattes; se todos os autores trabalhavam com o mesmo tema; se o estudo era multicêntrico; em qual revista o artigo foi publicado, o seu qualis e as bases de dados em que a revista estava indexada e se os descritores estavam adequados conforme a temática abordada.

A participação dos envolvidos foi satisfatória, porque eles não se restringiram ao fragmento proposto, lendo o artigo na íntegra. Isso fez com que fosse modificada a proposta metodológica do projeto, pois se previa o uso de um único artigo nos encontros virtuais, por meio da utilização de seções diferentes do mesmo. Todavia, devido ao interesse dos participantes em realizar a leitura do texto na íntegra para responder às questões, optou-se por utilizar um artigo diferente em cada encontro virtual.

Ainda, os participantes realizaram apontamentos críticos a respeito das fragilidades e das potencialidades na estrutura do artigo, além de compartilharem links de interesse para a identificação do Qualis da revista. Nesse sentido, o espaço também foi de troca de informações e esclarecimento de dúvidas. Houve posicionamentos diferentes e optou-se pela realização de um exercício crítico com as opiniões a respeito do artigo.

No encontro virtual B, contou-se também com a participação de 36 pessoas, sendo 11 profissionais de saúde e 25 acadêmicos. A partir do fragmento de um artigo, foram propostas algumas questões semelhantes ao primeiro encontro virtual, porém foram acrescentados outros itens referentes à introdução e à metodologia, tais como: os participantes deveriam verificar se a introdução era breve e objetiva, se apresentava a contextualização do tema com outros estudos já realizados, se oferecia ênfase às lacunas existentes e se justificava de forma científica a realização e a publicação da pesquisa.

Também foram avaliadas as referências apresentadas na introdução relacionadas ao tema de pesquisa, se a questão norteadora da pesquisa estava presente; se o objetivo estava claro e igual ao apresentado no resumo; se o método estava adequado à questão norteadora da pesquisa e ao objetivo proposto. Em caso de resposta negativa, solicitava-se a indicação de outros métodos de modo a dar conta do problema de pesquisa. Além disso, os participantes deveriam observar se o método do artigo estava descrito de forma clara, com todas as informações necessárias, se havia um referencial teórico ou metodológico e se o mesmo estava descrito de maneira que o leitor compreendesse. 
Curso de extensão de leitura crítica de artigos científicos: relato de experiência

Diante dos questionamentos, foram realizados comentários em relação ao conteúdo do artigo, mostrando a compreensão do tema e dos itens que deveriam ser contemplados em cada seção do artigo. Na ficha de avaliação desses dois encontros virtuais os participantes avaliaram a organização do grupo online como excelente ou boa; disparadores para discussão como excelente ou bom, sendo apontado apenas por um participante, como razoável; clareza na explicação das atividades como excelente ou boa e em relação às respostas das discussões realizadas em forma de comentários, grande parte avaliou como excelente ou boa.

No segundo encontro presencial, realizado no dia 28 de novembro, os participantes tiveram um momento de teorização, com temas considerados importantes pelos facilitadores, para a leitura de artigos científicos, dentre eles: caminhos para facilitar a busca de artigos em bases de dados, Qualis da Coordenação de Aperfeiçoamento de Pessoal de Nível Superior (CAPES), fator de impacto e índice H. Objetivou-se também abordar de forma aprofundada as seções que compõem um artigo científico e os itens essenciais para que esses sejam descritos de forma clara e adequada. Estiveram presentes 21 participantes, sendo seis profissionais de saúde e 15 acadêmicos. Quanto à avaliação, o encontro foi considerado satisfatório em termos de organização e conteúdo.

Os encontros virtuais C e D, realizados entre os dias 02 a 08 e 09 a 12 de dezembro, ocorreram entre o segundo e o terceiro presencial. No terceiro encontro virtual C foi utilizado como disparador um artigo de revisão integrativa, sendo usada a lista de critérios apresentada no segundo encontro presencial, de modo a favorecer a leitura crítica desses textos. Foram propostos nesse encontro os seguintes questionamentos: tipo de revisão que o artigo abordava; locais em que havia sido realizada a busca de artigos (bases de dados, bibliotecas virtuais); a estratégia utilizada para essa busca; os critérios de inclusão e exclusão que foram utilizados; quantos artigos foram encontrados na busca; quantos foram excluídos e o motivo; se os resultados abordavam realmente os dados dos artigos encontrados ou se era somente realizada a sua descrição; se o tema foi confrontado com a literatura, sendo realizada a discussão dos dados; a relevância, dentro do que se propunha, e se os resultados iam ao encontro do objetivo e da questão norteadora. Nesse encontro participaram 18 pessoas, sendo quatro profissionais e os demais acadêmicos.

No encontro virtual D participaram 31, oito profissionais de saúde e 23 acadêmicos. Como disparador, foi utilizado as considerações finais de um artigo, propondo ao participante analisar se havia articulação com o objetivo do artigo e com os resultados. Da mesma forma, os dois últimos encontros virtuais foram avaliados como excelente ou bom, nos itens já mencionados anteriormente. 
Curso de extensão de leitura crítica de artigos científicos: relato de experiência

No terceiro encontro presencial, realizado no dia 12 de dezembro, havia quatro profissionais e dez acadêmicos. Os 14 participantes foram divididos em pequenos grupos. A atividade proposta foi a leitura de um artigo com apontamentos críticos fundamentados no que foi discutido durante o curso. Além disso, as sugestões dadas foram no sentido de que o curso deveria ser ofertado para os alunos ingressantes na universidade, com o intuito de obterem uma aproximação e melhor compressão da estrutura de um artigo científico, pois entendem que isso facilitaria a sua leitura e aplicabilidade no contexto acadêmico.

\section{Discussão}

Um fato científico só é consolidado com a publicação. Entretanto, no contexto atual, há o aumento da competição para publicar, ao mesmo tempo que o número de artigos científicos submetidos às revistas extrapola o seu espaço disponível. Nessa disputa, os melhores textos têm maior probabilidade de serem escolhidos, sendo por isso necessária a produção de alta qualidade (PEREIRA, 2012a). Assim, quanto maior for a exigência na produção dos textos, provavelmente maior será sua qualidade e, consequentemente, sua leitura e divulgação.

Destaca-se que, na área da saúde, o profissional precisa se manter constantemente atualizado, em virtude dos avanços científicos e das mudanças tecnológicas. Assim, ele necessita saber ler e avaliar artigos científicos, a fim de ter acesso às informações verdadeiras e recentes (GUEDES; GUEDES, 2012). O leitor deve estar atento para selecionar as informações trazidas pelos artigos. Nesse entendimento, a proposta do curso surgiu de modo a aproximar a universidade da comunidade, buscando auxiliar acadêmicos e profissionais de saúde a ampliarem seu olhar quanto à leitura crítica de artigos científicos e a utilização dos resultados oriundos das investigações na qualificação da prática profissional.

Salienta-se que os artigos científicos constituem uma unidade de informação, devendo ser de domínio público. Por meio deles, as informações trazidas pelos autores são transformadas em conhecimento científico. Se o artigo é divulgado adequadamente, ele poderá ser lido, citado e utilizado por acadêmicos e profissionais de saúde em suas diversas atividades diárias (PEREIRA, 2012b).

Perante esse contexto, a realização dos encontros virtuais e o compartilhamento de artigos eletrônicos foram fatores que contribuíram para o andamento do curso. Ressalta-se que nos encontros virtuais não houve necessidade de todos os envolvidos estarem online, todavia, eles puderam fazer os comentários dentro do prazo estabelecido, que era de até sete dias depois da postagem. Estar offline e online no ciberespaço se confunde, pois estar perto e estar longe 
Curso de extensão de leitura crítica de artigos científicos: relato de experiência

passam a ter significados similares e diferentes bastando apenas um click no mouse para estar em comunicação com os outros (FÉLIX, 2012).

Outra potencialidade foi a utilização do recurso: criação de grupos no Facebook. Nesse espaço criado pode-se anexar arquivos, como materiais didáticos e fotos, realizar postagens, possibilitando que colaboradores e participantes curtam e comentem as atividades postadas. As redes sociais com base nos inúmeros recursos que a internet proporciona apresentam sua relevância, porém o desafio para os educadores é a incorporação dos recursos da internet em redes sociais com a finalidade de beneficiar o processo de ensino e aprendizagem (LORENZO, 2011).

Em outras palavras, os estudantes aprendem interagindo uns com os outros e construindo seu próprio entendimento. As múltiplas perspectivas oferecidas pela pedagogia narrativa baseada em comunidades virtuais aumentam esse processo e oferecem uma excelente oportunidade de aprendizado (WALSH; SOEREN, 2012). Conforme exposto, acredita-se que propostas como a do curso podem contribuir para o avanço no processo de ensino aprendizagem na área da saúde em virtude de sua concepção problematizadora, buscando transpor estratégias de ensino tradicionais que, muitas vezes, limitam a socialização e construção do conhecimento.

Apesar de se vivenciar uma era em que o conhecimento é gerado e compartilhado rapidamente, o leitor deve estar atento às informações veiculadas pelos artigos. Ter uma lista de pontos específicos a serem observados ajuda muito durante a realização da leitura. Entretanto, não basta seguir o roteiro, é preciso julgar com profundidade e com senso crítico (HOSSNE; VIEIRA, 2007). Este aspecto foi bastante discutido nos encontros, pois se espera que tanto os profissionais da área da saúde quanto os acadêmicos ampliem seu olhar, não apenas para a estrutura do artigo, mas principalmente para o conteúdo nele contido, o qual poderá ser aplicado na prática e qualificar a assistência em saúde.

Assim, desenvolver as habilidades de uma postura crítica quando se lê artigos científicos é um aspecto importante da instrução literária e interpretar textos com criticidade pode auxiliar as pessoas a se tornarem atentas às mensagens que são comunicadas. Elas aprendem como utilizar o pensamento crítico, ficam cautelosas quanto aos seus pontos de vista, isso influencia em suas interpretações dos textos e Conforme exposto, acredita-se que propostas como a do curso podem contribuir para o avanço no processo de ensino aprendizagem na área da saúde em virtude de sua concepção problematizadora, buscando transpor estratégias de ensino tradicionais que, muitas vezes, limitam a socialização e construção do conhecimento.interação com o meio social (LOBRON; SELMAN, 2007; STEVENS; BEAN, 2007). 
Curso de extensão de leitura crítica de artigos científicos: relato de experiência

Sendo assim, ao voltar-se o olhar para o cenário da saúde, reforça-se a necessidade de instrumentalizar os profissionais e acadêmicos para a leitura crítica de artigos científicos, visto que os resultados das investigações podem contribuir para o fortalecimento da atividade prática. Auxiliando, por exemplo, na construção de protocolos que respaldam as ações dos profissionais, além da adesão de novas formas de fazer pautadas no conhecimento científico.

Para compreender a linguagem científica é preciso analisar de forma crítica diversos aspectos durante a leitura do texto científico (Oliveira; Queiroz, 2012). Conforme já relatado, entre as atividades propostas pelo curso, apresentou-se uma lista de critérios para a leitura de qualidade de artigos científicos. Parte dessa lista é baseada no Strengthening the Reporting of Observational Studies in Epidemiology (STROBE), instrumento avaliativo de estudos epidemiológicos que foi traduzido por um grupo de pesquisadores brasileiros. O STROBE fornece recomendações sobre como relatar estudos de forma clara, com descrição precisa e completa (MALTA et al., 2010).

Assim, a adoção de uma lista de critérios, além de situar o leitor sobre o que deverá observar no artigo, permite que ele vá direto à seção que lhe interessa (OLIVEIRA; QUEIROZ, 2012). 0 conhecimento da estrutura é o elemento básico para compreender a composição de um artigo científico. Um roteiro com algumas dicas podem auxiliar o leitor a entender o conteúdo apresentado e a ter o domínio das características de cada parte proporcionando a apreciação da arte e da técnica relacionada à comunicação científica (PEREIRA, 2012a).

A composição da maioria dos artigos científicos inclui quatro seções, isto é, Introdução, Método, Resultados e Discussão (PEREIRA, 2012b; 2012c). As considerações finais ou conclusão aparecem em alguns. Geralmente, o leitor deve considerar alguns pontos chave, como na introdução, deve questionar sobre o que trata o estudo; na metodologia, como ele foi desenvolvido; nos resultados, quais as informações trazidas e, na discussão, averiguar se os resultados estão de acordo ou divergem de outros estudos realizados anteriormente (Pereira, 2011).

Ainda, havia sido solicitado aos participantes do curso buscar temas semelhantes aos discutidos nos artigos lidos, a fim de familiarizarem-se com as questões abordadas. Para isso, salientou-se a necessidade de procurar artigos publicados em periódicos científicos de qualidade. Essa tarefa consistiu em um aprendizado, por meio da revisão da literatura, contribuindo para o processo de reflexão (PEREIRA, 2013).

Também foi proposto aos participantes pesquisarem se os autores eram especialistas na temática do artigo, o que permitiu informar se o assunto abordado no texto foi desenvolvido por pessoas que possuem experiência sobre a área que está sendo discutida (OLIVEIRA; QUEIROZ, 
Curso de extensão de leitura crítica de artigos científicos: relato de experiência

2012). Muitas vezes, esse fato pode proporcionar maior credibilidade na informação apresentada.

De modo geral, acredita-se que a prática da leitura de artigos científicos pode auxiliar no desenvolvimento de uma visão crítica/reflexiva sobre o assunto abordado. As atividades propostas pelo Curso de Extensão de Leitura Crítica de Artigos Científicos visaram instrumentalizar os participantes quanto à leitura de textos científicos, na busca de informações que possam favorecer tanto no conhecimento como na prática diária de trabalho. A proposta implantada almejou também o fortalecimento do hábito da leitura, o qual deve ser compreendido como um momento prazeroso, permitindo ampliar o olhar sobre os pontos importantes que devem ser considerados para a boa qualidade de um artigo científico.

Os exercícios de prática da leitura crítica de artigos promovidos pelo curso de extensão tiveram o objetivo de fortalecer nos participantes a compreensão de critérios importantes que devem ser analisados em um texto. Deste modo, o fato de ler e de interpretar constituem práticas necessárias para a geração do conhecimento. É de suma importância que as pessoas desenvolvam habilidades de leitura, buscando por meio da seleção, compreensão e interpretação das informações presentes em textos científicos da área de interesse, uma visão crítica do respectivo assunto (BONA; DAMINELLI; OLIVEIRA, 2012). Mediante a ampliação do olhar crítico dos leitores, esse processo instigado pelo curso poderá contribuir também à atenção que os autores devem adotar durante a construção dos seus artigos, visando assim à qualidade na produção científica.

\section{Considerações finais}

A proposta do Curso de Extensão de Leitura Crítica de Artigos Científicos era proporcionar um espaço para discussão sobre a leitura de artigos científicos da área de saúde junto a estudantes universitários e profissionais de saúde. Ainda, se objetivou socializar os critérios que auxiliam na leitura crítica e na escolha de artigos científicos, além de discutir sobre a importância de relacionar a produção cientifica com a prática, de modo a qualificá-la.

Ao analisar a experiência relatada, pode-se afirmar que os objetivos foram atingidos, pois o espaço propiciado durante o curso, na forma presencial ou virtual, permitiu a troca de conhecimentos e de informações, mostrando o desenvolvimento satisfatório em relação à leitura crítica apresentada pelos participantes. Esses, ainda consideraram que, com o curso, aprenderam a analisar o conteúdo, bem como identificar fragilidades e potencialidades de um artigo, para escolhê-lo e utilizá-lo em seus espaços de estudos ou ocupacionais. 
Curso de extensão de leitura crítica de artigos científicos: relato de experiência

Diante do cumprimento das metas estabelecidas e dos resultados gerados, associados à discussão trazida neste relato de experiência, é possível mostrar o quanto a formação de um espaço de leitura, de análise e de reflexão sobre a composição dos artigos científicos torna-se relevante para acadêmicos e profissionais de saúde, além dos colaboradores do projeto que implantaram o curso de extensão.

O desenvolvimento produtivo das atividades estabelecidas, visualizado pelo alcance dos objetivos iniciais, pode favorecer à continuidade do Curso de Extensão de Leitura Crítica de Artigos Científicos, por meio da abertura de novas edições, ampliando assim a participação de mais pessoas, buscando instrumentalizar tanto os acadêmicos como os profissionais inseridos nos serviços de saúde.

\section{Referências}

BERTOLUCI, Kaluana Nunes. Letramento acadêmico: leitura (s) de um curso de pedagogia. Revista Ao Pé da Letra, Recife, v.11, n.2, p. 105-124, 2009.

BONA, Aline Silva; DAMINELLI, Elisa; OLIVEIRA, Humberto Luz. Aprendendo a ler artigo científico. Revista Trajetória Multicursos - FACOS/CNEC, Osório, v. 5, n. 6, p.20-35, jul. 2012.

BROFMAN, Paulo Roberto. A importância das publicações científicas. Cogitare Enfermagem, Curitiba, v. 17, n. 3, p. 419-421, 2012.

CARDOSO, Giane Carrera.; PELOZO, Rita de Cássia Borguetti. A importância da leitura na formação do indivíduo. Revista Científica Eletrônica de Pedagogia, São Paulo, v. 5, n. 9, p. 1-7, 2007.

CAVALCANTE, Bruna Luana de Lima; LIMA, Uirassú Tupinambá Silva de. Relato de experiência de uma estudante de enfermagem em um consultório especializado em tratamento de feridas. Journal of Nursing and Health, Pelotas, v.1, n.2, p.94-103, jan/jun 2012.

FÉLIX, Jeane. Entrevistas on-line ou algumas pistas de como utilizar bate-papos virtuais em pesquisas na educação e na saúde. In: MEYER, Dagmar Edtermann; PARAÍSO, Marlucy. A (org). Metodologias de pesquisas pós-críticas em educação. Belo Horizonte: Mazza Edições, 2012. p. 131-152.

GUEDES, Hermila Tavares Vilar; GUEDES, Jorge Carvalho. Avaliação, pelos estudantes, da atividade "trabalho de conclusão de curso" como integralização do eixo curricular de iniciação à pesquisa científica em um curso de medicina. Revista Brasileira de Educação Médica, Manguinhos, v.36, n.2, p.162-171, 2012.

HOSSNE, William Saad; VIEIRA, Sonia Vieira. Fraude em ciência: onde estamos? Revista Bioética, Brasília, v.15, n.1, p.39-47, 2007.

JANKS, Hilary. Literacy and power. New York: Routledge, 2010. 
Curso de extensão de leitura crítica de artigos científicos: relato de experiência

JEZINE, Edineide Mesquita. Multiversidade e Extensão Universitária in. FARIA, Dóris Santos de (org). Construção Conceitual da Extensão na America Latina. Brasília: UNB, 2001.

LOBRON, Alisson; SELMAN, Robert. The interdependence of social awareness and literacy Instruction. The Reading Teacher, Newrak, v.60, n.6, p. 528-537, 2007.

LORENZO, Eder. A utilização das Redes Sociais na Educação. [S.I.]: Books Google, 2011. Disponível em: $<$ http://books.google.com.br/books?id=PGk3AgAAQBAJ\&pg=PA264\&dq=0+uso + do + facebook + na+educa\%C3\%A7\%C3\%A3o+virtual\&hl=ptBR\&sa=X\&ei=E8jKU63cMZHKsQTd_oG4DQ\&ved=0CCEQ6AEwAQ\#v=onepage\&q\&f=false >. Acesso em: 19 jul. 2014.

MATENCIO, Maria de Lourdes Meirelles. Atividades de retextualização em práticas acadêmicas: um estudo do gênero resumo. Scripta (PUCMG), Belo Horizonte, v. 6, n. 11, p. 25$32,2002$.

OLIVEIRA, Jane Raquel Silva de.; QUEIROZ, Salete Linhares. A retórica da linguagem científica: das bases teóricas à elaboração de material didático para o ensino superior de Química. Química Nova, São Paulo, v.35, n.4, p. 851-857, 2012.

PEREIRA, Maurício Gomes. Artigos científicos: como redigir, publicar e avaliar. Rio de Janeiro: Guanabara-Koogan, 2011.

PEREIRA, Maurício Gomes. Preparo para a redação do artigo científico. Epidemiologia e Serviços de Saúde, Brasília, v.21, n.3, p.515-516, set. 2012a.

PEREIRA, Maurício Gomes. Estrutura do artigo científico. Epidemiologia e Serviços de Saúde, Brasília,v.21, n.2, p.351-352, jun. 2012b.

PEREIRA, Maurício Gomes. A introdução de um artigo científico. Epidemiologia e Serviços de Saúde, Brasília, v.21, n.4, p.675-676, 2012c.

PEREIRA, Maurício Gomes. A seção de discussão de um artigo científico. Epidemiologia e Serviços de Saúde, Brasília, v.22, n.3, p.537-538, set. 2013.

RAMOS, Amauri Pereira; CARVALHO, José Oscar. A utilização de ambientes virtuais para a colaboração por grupos de pesquisa brasileiros: uma análise do desenvolvimento de trabalhos de maneira colaborativa. Revista de Ciência da Informação, Rio de Janeiro, v. 8 n. 1, 2007. Disponível em: <http://www.dgz.org.br/fev07/F_I_art.htm>. Acesso em: 30 maio 2014.

ROCHA, Gurgel. A Construção do Conceito de Extensão universitária na America Latina. In. FARIA, Dóris Santos de (org). Construção Conceitual da Extensão na America Latina. Brasília: UNB, 2001.

SERRANO, Rossana Marília Souto Maior. Conceitos de extensão universitária: um diálogo com Paulo Freire. Disponível em: <http://www.prac.ufpb.br/copac/extelar/atividades/discussao/artigos/conceitos_de_extensao_ universitaria.pdf $>$. Acesso em: 30 mar. 2014.

SOUZA, Aana Luiza Lima. A história da extensão universitária. 2ª ed. São Paulo: Alínea, 2010. 
Curso de extensão de leitura crítica de artigos científicos: relato de experiência

STEVENS, Lisa; BEAN, Thomas. Critical literacy: Context, research, and practice in the K-12 classroom. Thousand Oaks: Sage, 2007.

UFPEL. Resolução no 04 de 21 de março de 2013. Revoga a Resolução n. 10, de 9 nov. 2006, que dispõe sobre o Regulamento Geral das Atividades Extensionistas e Culturais na Universidade Federal de Pelotas- UFPEL, e dá outras providencias. Disponível em: <http://wp.ufpel.edu.br/prec/files/2010/05/Resolu\%C3\%A7\%C3\%A3o-COCEPE-042013.pdf>. Acesso em: 15 de jun 2015.

VALERIO, Palmira Moriconi; PINHEIRO, Lena Vania Ribeiro. Da comunicação científica à divulgação. TransInformação, Campinas, v. 20, n. 2, p. 159-169, 2008.

WALSH, Martin; SOEREN, M. Interprofessional learning and virtual communities: An opportunity for the future. Journal of Interprofessional Care, London, v.26, p.43-48, 2012.

Critical reading extension course of scientific articles: experience report

\begin{abstract}
It was aimed to report the experience about the Extension Course on Critical Reading of Scientific Articles, which enabled a space for discussion of readings and interpretation of articles on health sciences, within students and health professionals. In this project, seven meetings were performed: three happened in person, and had discussion of an article for each subgroup, and four meetings happened virtually, which touched off discussions online. The Course enabled the instrumentalization of health professionals and students to critical readings of scientific articles, which might be applied to exercise and qualify the health assistance.
\end{abstract}

\section{Keywords}

Journal Article. Community-Institutional Relations. Extension Activity.
Curso de extensión de lectura crítica de artículos científicos: relato de experiencia

\section{Resumen}

Se objetivó relatar la experiencia del Curso de Extensión de Lectura Crítica de Artículos Científicos, que posibilitó un espacio para discusión de lectura e interpretación de artículos de la área de la salud junto a académicos y profesionales de salud. En este proyecto, fueron realizados siete encuentros, siendo tres presenciales, con discusión de un artículo por subgrupo, y cuatro virtuales, con disparadores para discusión en una red social. Se destaca que el Curso posibilitó la instrumentalización de profesionales de la salud y académicos para lectura crítica de artículos científicos, que podrá ser aplicado en la práctica y cualificar na asistencia en salud.

\section{Palabras clave}

Artículo de revista. Relaciones ComunidadInstitución. Actividad de Extensión.

Original submetido em: 11 maio 2015

Aceito para publicação em: 8 jun. 2015 
Sobre as autoras:

\section{Stefanie Griebeler Oliveira}

Enfermeira. Doutora em Enfermagem pela Universidade Federal do Rio Grande do Sul (UFRGS). Professora da Faculdade de Enfermagem da Universidade Federal de Pelotas (UFPel).

\section{Simone Coelho Amestoy}

Enfermeira. Doutora em Enfermagem. Professora do Departamento e do Programa de Pós-Graduação em Enfermagem da Universidade Federal de Pelotas (UFPel). Membro do Grupo de Pesquisa em Educação em Enfermagem e Saúde e do Núcleo de Estudos e Pesquisas em Enfermagem.

\section{Raquel Pötter Garcia}

Enfermeira. Mestre em Enfermagem. Doutoranda em Ciências pelo Programa de Pós-Graduação em Enfermagem, Universidade Federal de Pelotas (UFPel). Professora da Universidade Federal do Pampa (Unipampa).

\section{Bianca Pozza dos Santos}

Mestre em Ciências. Doutoranda pelo Programa de Pós-Graduação em Enfermagem da Universidade Federal de Pelotas (UFPel)

\section{Patricia Mirapalheta Pereira}

Enfermeira. Mestre e Doutoranda em Ciências da Saúde pelo Programa da Pós Graduação em Enfermagem da Universidade Federal de Pelotas (UFPel). Bolsista da Coordenação de Aperfeiçoamento de Pessoal de Nível Superior (CAPES).

\section{Andrieli Daiane Zdanski de Souza}

Mestre em Enfermagem pela Universidade Federal de Pelotas (UFPel). Enfermeira Intensivista do Hospital Ulbra/Mãe de Deus.

\section{Julia Cardoso Parra}

Acadêmica de Enfermagem na Universidade Federal de Pelotas (UFPel). Bolsista PROEXT no Projeto $O$ empoderamento de mulheres frente ao aleitamento materno: proposta de efetivação de políticas públicas voltadas à promoção da saúde materno-infantil.

\section{Silvia Francine Sartor}

Graduanda Sanduíche no Medical Sciences, Indiana University Bloomington (EUA) e Universidade Federal de Pelotas (UFPel).

\section{Kimberly Larroque Velleda}

Acadêmica de Enfermagem na Universidade Federal de Pelotas (UFPel). 